recently antibodies to human muscle have been detected in the serum of some patients with myasthenia gravis (Strauss et al., 1960 ; Beutner et al., 1962 ; van der Geld et al., 1963). White and Marshall (1962) have also demonstrated antinuclear factor in the serum of six out of 16 patients with myasthenia gravis. A close correlation between these serological observations and clinical manifestations has not yet been described, and it would be difficult to account for the reported improvement after dialysis (Stricker et al., 1960), as it is unlikely that antibodies could be cleared from the circulation.

Some of the clinical features of neonatal myasthenia gravis, particularly its duration, its complete remission, and its tendency to affect consecutive infants, are compatible with the suggestion (Beutner et al., 1962) that the disease might represent the passive transfer of maternal antibodies. The clinical details of our cases could be interpreted in accordance with this suggestion, but the available serological information offers no confirmation of transfer of antibodies. It appears unlikely that muscle antibodies of antinuclear factor can be directly related to the neonatal disorder. In Case 1 complement-fixation tests and an indirect immunofluorescent technique failed to detect any circulating antibodies to normal human muscle. Antinuclear factor may have crossed from the maternal circulation to the foetus in Case 1, but it was absent in the serum of Case 2.

Until further observations, possibly utilizing more sensitive serological methods, correlate the passage of antibodies from mother to child with clinical disturbance in the infant, the view that neonatal myasthenia gravis is the manifestation of passive transfer of maternal antibodies must remain speculative and unproved.

\section{Summary}

Two cases of transient neonatal myasthenia gravis are reported. In the first case circulating antibodies to muscle could not be detected, but antinuclear factor was present in the serum of mother and child. Antinuclear factor was not present in the serum of the second infant.

The disorder may be more common than the literature suggests. The potential gravity of the unrecognized illness and the favourable therapeutic response to adequate doses of parenteral anticholinesterases are stressed. The condition may affect the children of untreated myasthenic mothers. Maternal medication probably influences the delay before symptoms are apparent in the child.

Current views on the nature of myasthenia gravis are reviewed. The available serological information in our cases does not confirm the hypothesis that transient neonatal myasthenia gravis represents the passive transfer of maternal antibodies.

We are grateful to the physicians and surgeons concerned for permission to publish cases under their care, and to the Muscular Dystrophy Group, who aided this work with a grant for secretarial assistance.

\section{REFERENCES}

Beutner, E. H., Witebsky, E., Ricken, D., and Adler, R. H. (1962). F. Amer. med. Ass., 182, 46 .

Churchill-Davidson, H. C., and Wise, R. P. (1963). Anaesthesiology, 24, 271.

Fraser, D., and Turner, J. W. A. (1953). Lancet, 2, 417.

Kibrick, S. (1954). Pediatrics, 14, 365.

Nastuk, W. L., Plescia, O. J., and Osserman, K. E. (1960). Proc. Soc. exp. Biol.' (N.Y.), 105, 177 .

Simpson, J. A. (1960). Scot. med. F., 5, 419.

Strauss, A. J., Seegal, B. C., Hsu, K. C., Burkholder, P. M. Nastuk, W. L., and Osserman, K. E. (1960).' Proc. Soc. exp. Biol. (N.Y.), 105, 184.

Stricker, E., Thölen, H., Massini, M. A., and Staub, H. (1960). F. Neurol. Neurosurg. Psychiat., 23, 291.

Strickroot, F. L., Schaeffer, R. L., and Bergo, H. L. (1942). F. Amer. med. Ass., 120, 1207.

van der Geld, H., Feltkamp, T. E. W., van Loghem, J. J., Oosterhuis, H. J. G. H., and Biemond, A. (1963). Lancet, 2, 373.

Viets, H. R., and Brown, M. R. (1951). New Engl. 7. Med., 245, 647.

Walker, M. B. (1938). Proc. roy. Soc. Med., 31, 722.

White, R. G., and Marshall, A. H. E (1962). Lancet, 2, 120

Wilson, A., and Stoner, H. B. (1944). Quart. F. Med., 13, 1.

\footnotetext{
* Associate Professor of Pharmacy and Biopharmaceutics, School of Pharmacy, State University of New York, Buffalo 14, N.Y., U.S.A. † Associate Chief of Staff, Vererans Administration Hospital, Palo Alto, Calif., U.S.A.
}

frequency of the maintenance doses can be determined only if the rate of elimination of the drug is known (Levy, 1963).

Surprisingly, data concerning the kinetics of elimination of salicylates by humans are very sparse, despite the long history and wide use of these drugs. It was thought desirable, therefore, to determine the rate of elimination of salicylate in a group of subjects under conditions which permitted evaluation of both intersubject and intrasubject variations.

\section{Experimental}

Seventeen healthy human subjects, consisting of seven men and ten women, aged 23 to 53 , weighing 52 to $96 \mathrm{~kg}$., were given $1 \mathrm{~g}$. (three 0.33 -g. tablets) of aspirin on an empty stomach. Blood samples were taken two, four, six, eight, and in most cases also 10 hours after drug administration. Plasma salicylate concentrations were determined by the colorimetric method of Trinder (1954). After one week the test was repeated. 


\section{Results and Discussion}

Individual plasma salicylate concentrations were found to decline exponentially as predicted by theory ; from these data individual first-order elimination rate constants ${ }^{1}$ were determined. In 12 subjects the rate constants obțained in two tests differed by less than $20 \%$ for any one individual, while somewhat greater differences were found in the other five subjects (see Chart). On the basis of the elimination rate constants obtained from only the 12 subjects who yielded closely reproducible values, averages and ranges were calculated for the group as well as separately for the male and female subjects (see Table). There was no apparent difference in elimination rate between sexes and no apparent correlation between elimination rate and weight or age. However, the significance of these conclusions is limited by the small number of subjects studied.

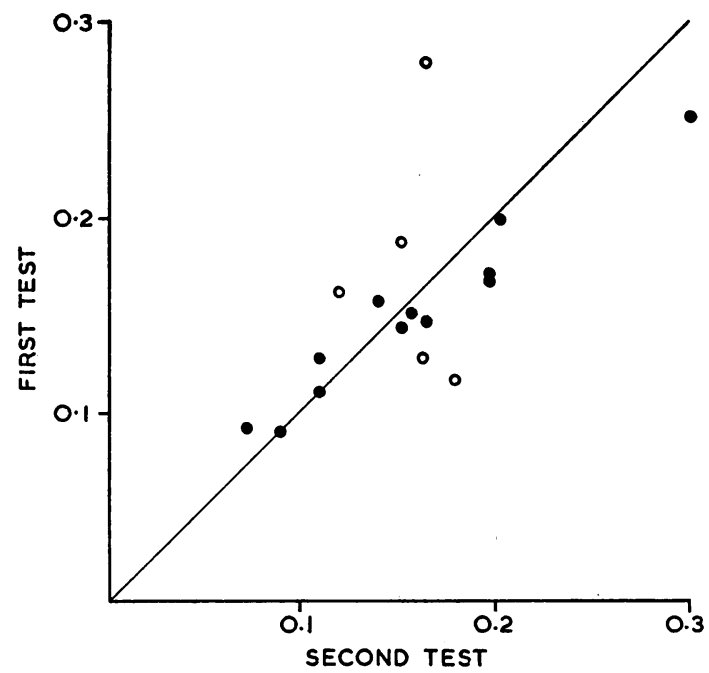

Plot of individual elimination rate constants for salicylate (expressed in reciprocal hours), determined twice (one week apart) in 17 adult humans. Line (slope 1.0) indicates position of points if correlation between tests had been position of points if correlation between tests had been perfect. tests ; O, rate constants which differ by $20 \%$ or more.

\begin{tabular}{|c|c|c|c|}
\hline \multirow{2}{*}{ No. of Subjects } & \multirow{2}{*}{ Sex } & \multicolumn{2}{|c|}{ Elimination-rate Constant $\left(\mathrm{hr} .^{-1}\right)$} \\
\hline & & Harmonic Mean & Range \\
\hline $\begin{array}{r}4 \\
8 \\
12\end{array}$ & $\begin{array}{l}\text { Male } \\
\text { Female } \\
\text { Male and female }\end{array}$ & $\begin{array}{l}0.160 \\
0.130 \\
0.138\end{array}$ & $\begin{array}{l}0 \cdot 148-0 \cdot 201 \\
0.082-0 \cdot 274 \\
0.082-0 \cdot 274\end{array}$ \\
\hline
\end{tabular}

* Determined from drug-plasma levels after oral administration of $1 \mathrm{~g}$. aspirin. Determined from drug-plasma levels after oral administration of 1 g. aspirin
+ Data based on rate constants which are the averages of two tests per subject.

The marked intersubject variation of salicylate-elimination rates is very significant. Expressed in terms of biological half-life $\left(t_{0.5}\right.$, the time necessary to reduce body drug content by $50 \%$ ), values ranged from 2.55 to 8.5 hours. It is likely that studies of a larger number of subjects would reveal even greater differences in salicylate-elimination rates. The possible implications of these differences between subjects can be illustrated by considering the amount of salicylate in the body immediately after administration of one loading dose (D*) and as few as three maintenance doses (D). Based on the average salicylate half-life found in the group (5.0 hours), one may administer a loading dose $\mathrm{D}^{*}$ followed by maintenance doses of half that size $\left(D=0.5 D^{*}\right)$ given at five-hour intervals. ${ }^{2}$ Assuming that the drug is administered in rapidly absorbed

1 The elimination rate constant $\mathrm{K}$, when expressed in reciprocal hours, designates the fraction of body drug content eliminated per hour. It is related to half-life $\left(t_{0.8}\right)$ as follows: $K=0.693 / t_{0.8}$.

2 This regimen (maintenance doses half the size of the loading dose, given at intervals equal to the biological half-life) has been shown to be ideal in many cases, on the basis of theoretical considerations (Krüger-Thiemer, 1960). form, the body salicylate content in a subject who eliminates the drug at the observed average rate (elimination-rate constant $\left.0.138 \mathrm{hr}^{-1}\right)$ is about $\mathrm{D}^{*}$ shortly after administration of the third maintenance dose. In other words, the body drug content is maintained at its initial (and desired) level. On the other hand, the same dosage schedule, if used for the most rapid salicylate eliminator in the group studied, would yield a body salicylate content of only about $0.67 \mathrm{D}^{*}$. Given to a subject who eliminates salicylate at the lowest rate found in this study, the average dosage regimen described above would result in a body salicylate content of about $1.4 \mathrm{D}^{*}(40 \%$ greater than the loading dose) after the third maintenance dose.

These examples illustrate the need to adjust salicylate dosage regimens individually on the basis of a subject's predetermined elimination rate if the incidence of therapeutic failure (due to subtherapeutic drug levels) or toxic effects (due to drug accumulation) is to be minimized. As pointed out previously (Levy, 1963), there are a number of factors which can cause changes in salicylate-elimination kinetics during disease. These changes make it desirable, during intensive salicylate therapy, to re-determine the elimination-rate constant periodically, especially if the patient's condition, treatment, or diet has changed appreciably. The appearance of side-effects (such as tinnitus), indicative of overdosage due to drug accumulation, may call for a change in dosing intervals rather than in amount of drug per dose, if therapeutically adequate drug concentrations are to be maintained at all times. Methods for the calculation of appropriate dosing regimens based on considerations of various pharmacokinetic parameters have been described (KrügerThiemer, 1960).

Only a few other studies of salicylate-elimination rates in humans are available. Brodie et al. (1959) reported an average half-life of 6.1 hours and range of 4.7 to 9.0 hours in six normal subjects given $1.3 \mathrm{~g}$. of salicylic acid intravenously. Apparently, only one determination of elimination rate was made in each subject, and thus the possible effect of relatively high urine $p H$, or other factors which can occasionally modify individual elimination rates, was not excluded. However, the reported values are in general agreement with those found in the present study.

Evans (cited by Evans and Clarke, 1961) has measured serum salicylate concentrations in 100 subjects three hours after drug administration. His purpose was to determine if there are genetic variations (polymorphism) in salicylate elimination. While the study revealed marked intersubject differences in serum salicylate levels these cannot be ascribed solely to variations in elimination rate. They can also be due, totally or in part, to individual differences in drug-absorption rates (Nelson, 1963) or apparent volumes of distribution.

Elimination of salicylate after ingestion of toxic amounts (Done, 1960) is significantly slower than after administration of therapeutic doses such as the 1-g. dose given in the present study. This appears to be due to limitations of conjugating capacity, resulting in decreased biotransformation of the drug (Levy, 1964). Animal experiments suggest that prolonged salicylate administration has no effect on elimination kinetics (Burns, 1964).

Preliminary indications of the apparently biochemical individuality of humans with respect to salicylate biotransformation have already been described (Levy and Gagliardi, 1963), and studies are currently in progress to elucidate the biochemical and/or physiological mechanism responsible for intersubject variations in salicylate-elimination rate.

\section{Summary}

Salicylate-elimination rate, studied in a group of healthy adult men and women, was found to vary appreciably between 
subjects. It is desirable that the dosage schedule in intensive salicylate therapy be adjusted individually on the basis of the patient's predetermined salicylate elimination rate in order to prevent drug accumulation or gradual decline of drug concentration to subtherapeutic levels.

\section{REFERENCES}

Brodie, B. B., Burns, J. J., and Weiner, M. (1959). Med. exp. (Basel), 1, 290.
Burns, J. J. (1964). Paper presented at Conference on Evaluation and Mechanisms of Drug Toxicity, N.Y. Academy of Science, March 5-7, New York.

Done, A. K. (1960). Pediatrics, 26, 800

Evans, D. A. P., and Clarke, C. A. (1961). Brit. med. Bull., 17, 234. Krüger-Thiemer, E. (1960). Klin. Wschr., 38, 514.

Levy, G. (1963). In The Salicylates, An International Symposium, p. 9. Churchill, London.

(1964). In Prescription Pharmacy, edited by J. B. Sprowls, p. 47. Lippincott, Philadelphia.

Lippincott, Philadelphia.

- and Gagliardi, B. A. (1963). 7. pharm. Sci., 52, 730

Nelson, E. (1963). F. theor. Biol., 5, 493.

Talbott, J. H. (1960). In Drugs of Choice 1960-1961, edited by W. Modell, p. 599. Mosby, St. Louis.

Trinder, P. (1954). Biochem. f., 57, 301.

\title{
Quinethazone, a new Oral Diuretic
}

\author{
GERALD SANDLER,* M.D., M.R.C.P.
}

Quinethazone (Aquamox) is a recently introduced oral diuretic agent in which a cyclic carbamyl group has replaced the cyclic sulphamyl group present in the thiazides. It therefore differs basically from the thiazide diuretics in the absence of sulphur-1, 1-dioxide ring system from the benzothiadiazine molecule (Fig. 1).<smiles>CCC1NC(=O)c2cc(S(N)(=O)=O)c(Cl)cc2N1</smiles>
QUINETHAZONE<smiles></smiles>

CHLOROTHIAZIDE
FIG. 1.-Chemical structure of quinethazone and chlorothiazide.

Several studies have established its effectiveness, which is similar to that of chlorothiazide (Ford, 1962 ; Seller et al., 1962). Preliminary experimental observations suggested also that when quinethazone is used in combination with acetazolamide the natriuretic effect is enhanced but the urinary loss of potassium is reduced (Herken and Senft, unpublished observations, 1962).

It is the purpose of the present study to investigate the effectiveness of quinethazone as an oral diuretic both in normal subjects and in patients with congestive cardiac failure, and also to assess the effect of the combination of quinethazone and acetazolamide on the urinary loss of sodium and potassium in these patients.

\section{Patients and Methods}

\section{Studies in Normal Subjects}

The diuretic effect of quinethazone and acetazolamide alone and in combination was studied in four healthy adults (three male, one female) aged 25 to 27 . During the investigations the subjects carried out normal hospital duties and took a normal diet with unrestricted fluid. The dose of quinethazone was $200 \mathrm{mg}$. and acetazolamide $500 \mathrm{mg}$. The drugs were given at 8 a.m., and subsequently two-hourly collections of urine were made for 10 hours. On the day preceding administration of each drug similar urinary collec-

\footnotetext{
*From the Royal Infirmary, Sheffield.
}

tions were made to provide control levels of urinary volume and electrolyte excretion. A period of two days was allowed between administration of the different drugs. The order of the initial drug was varied so that each of three subjects respectively received quinethazone, acetazolamide, and a combination of quinethazone and acetazolamide to start. In addition these three subjects received chlorothiazide, 1 g., for comparison with the other drugs.

In the fourth subject fludrocortisone was used to increase urinary potassium excretion and so allow investigation of the effect of quinethazone and acetazolamide in inhibiting loss of potassium. Initially, fludrocortisone was given in a dose of $1.5 \mathrm{mg}$. at night and $1 \mathrm{mg}$. at $8 \mathrm{a} . \mathrm{m}$. the next day, and twohourly urine collections were made for 10 hours to provide control values of sodium and potassium excretion. Subsequently the same regime was adopted together with quinethazone alone and in combination with acetazolamide given with the morning dose of fludrocortisone. On each occasion a similar control collection was made on the day preceding drug administration. The volume of each specimen of urine was recorded and the content of sodium, potassium, and chloride estimated.

\section{Clinical Studies}

Clinical studies were carried out on 16 patients, and these were subdivided into three groups :

Group 1.-Six patients with rheumatic heart disease-four of whom had chronic congestive cardiac failure, and two who had never had cardiac failure (these two patients were being treated for subacute bacterial endocarditis at the time of study). The effects of single doses of $200 \mathrm{mg}$. of quinethazone and $500 \mathrm{mg}$. of acetazolamide, alone and in combination, were studied in this group.

Group 2.-Four patients with congestive cardiac failure due to rheumatic heart disease or cor pulmonale in whom quinethazone and acetazolamide were compared with chlorothiazide ( $1 \mathrm{~g}$. dose).

Group 3.-Four out-patients with chronic congestive cardiac failure due to rheumatic heart disease or cor pulmonale in whom the effects of prolonged courses of quinethazone, $200 \mathrm{mg}$. daily, acetazolamide, $500 \mathrm{mg}$. daily, or chlorothiazide, $1 \mathrm{~g}$. daily, were investigated.

In groups 1 and 2 the drugs were given at 10 a.m. and urine was collected for 24 hours from this time and assessed for volume and content of sodium, potassium, and chloride. 